\title{
Ultrasound elasticity imaging using Golay code
}

\author{
Hui Peng ${ }^{a}$, Juhong Tie \\ School of Software Engineering, Chengdu University of Information Technology, Chengdu, China
}

\begin{abstract}
Ultrasound elasticity imaging is becoming a new diagnostic tool for clinicians to detect lesions or cancers in tissues. In this paper, Golay code is applied to elasticity imaging to improve its quality. Phase-zero algorithm is employed as the displacement estimator, and the amplitude modulation correction location estimate method is used as the location estimator. We compared the performance of Golay code and the conventional short pulse in simulation method. The simulation results demonstrate that Golay code can achieve higher elastographic signal-to-noise ratio (SNRe) than the short pulse in low echo signal-to-noise ratio (eSNR) conditions, because the eSNR gain with Golay code increases the accuracy of the displacement estimates. However, in high eSNR conditions, Golay code performs worse than the short pulse, because the range sidelobe level of Golay code will decrease the SNRe and the performance of Golay code depends mainly on its range sidelobe level in high eSNR conditions. Therefore, the optimal conditions for Goaly code to be used in elasticity imaging are the low eSNR, great depth or high attenuation conditions.
\end{abstract}

\section{Introduction}

Ultrasound elasticity imaging is becoming a new diagnostic tool for clinicians to detect lesions or cancers in tissues. However, in some practical ultrasonic elasticity imaging systems, poor quality of strain images degrades its value of clinical application [1]. One of major quality metrics of strain images is elastographic signal-to-noise ratio (SNRe) affected by many factors, such as echo signal-tonoise ratio (eSNR), applied strain, attenuation, etc. Many methods [2-6] were proposed to improve the quality of ulrasound strain imaging.

O'Donnell [7] predicted that the transmitted energy can be increased up to $15-20 \mathrm{~dB}$ by coded excitation before reaching particular intensity limits (Isppa, Ispta). The additional eSNR can be used to improve the performance of elasticity imaging. Jie Liu et al. [4] first applied coded excitation to ultrasonic strain imaging. Reference [5] studied the effects of various factors on chirp-coded strain imaging. In this paper, we applied Golay codes to elasticity imaging to improve its quality. We investigated the design considerations for Goaly-coded strain imaging, and compared the performance of Golay code with the conventional short pulse in different eSNR conditions in simulation method. The optimal condition for Golay code to be used in strain imaging is discussed.

\footnotetext{
${ }^{a}$ Corresponding author: penghui@cuit.edu.cn
} 


\section{Method}

\subsection{Golay code}

Golay codes are pairs of bi-phase modulation codes which consist of two sequences of the same length $\mathrm{N}$ whose auto-correlation functions have sidelobes equal in magnitude but opposite in sign. Summing them up results in a composite auto-correlation function with a peak of $2 \mathrm{~N}$ and zero sidelobes [8]. A pair of bi-phase sequences $A(a(n), n=1, \ldots, N)$ and $B(b(n), n=1, \ldots, N)$ of length $\mathrm{N}$ is a Golay code if, and only if, it satisfies [9]:

$$
a(n) * a(-n)+b(n) * b(-n)=2 N \delta(n)
$$

which is known as the complementarity condition.

Fig. 1 shows the decoding process of Golay codes of length 4 using matched filter. It can be seen from Fig.1 that Golay codes can cancel perfectly the range sidelobes after summing the matched filter outputs up and the amplitude of the output sequence is $2 \mathrm{~N}$ times of that of Golay codes.

Unlike the available longest Baker code which length is 13, Golay pairs of any length can be constructed recursively from other Golay pairs. Given a Golay pair A, B, another Golay pair of twice the length can be generated by $[A B, A(-B)]$. The recursion can be initiated with a Golay code of length 2: $\mathrm{A}=[1,1]$ and $\mathrm{B}=[1,-1]$.

Golay codes cannot be transmitted directly, and they need be modulated by a base pulse sequence in order to achieve better spectral control and pulse shaping as well as greater robustness to motion for multitransmission code [9]. The encoding process of the Golay-code-modulated base sequence can be described as a convolution of base pulse sequence with an oversampled Golay code sequence:

$$
\begin{aligned}
& x_{a}(n)=\hat{a}(n) * s(n) \\
& x_{b}(n)=\hat{b}(n) * s(n)
\end{aligned}
$$

where $\hat{a}(n)$ and $\hat{b}(n)$ are a pair of oversampled Golay sequences; $s(n)$ is the base pulse sequence; $x_{a}(n)$ and $x_{b}(n)$ are a pair of Golay-code-modulated base sequences which are transmitted; and $*$ is the convolution operator.

The received Golay-code-modulated sequences are often decoded by the matched filter to achieve maximum eSNR and same axial resolution of the base pulse sequence [10]. The decoding process of the received Golay-code-modulated sequences can be expressed as the formula (3):

$$
\begin{aligned}
y(n) & =x_{a}(n) * \hat{a}(-n)+x_{b}(n) * \hat{b}(-n) \\
& =\hat{a}(n) * s(n) * \hat{a}(-n)+\hat{b}(n) * s(n) * \hat{b}(-n) \\
& =[\hat{a}(n) * \hat{a}(-n)+\hat{b}(n) * \hat{b}(-n)] * s(n) \\
& =2 \mathrm{~N} s(n)
\end{aligned}
$$

where $y(n)$ is the output pulse sequence that is the sum of a pair matched filter outputs. It can be seen from the above formula (3) that the amplitude of the output pulse sequence is $2 \mathrm{~N}$ times of that of the base pulse sequence, and the output pulse sequence is without any range sidelobes. In practice, the displacement motion of scatters during two transmission of a pair of Golay codes will produce the range sidelobes of the output pulse sequence. 


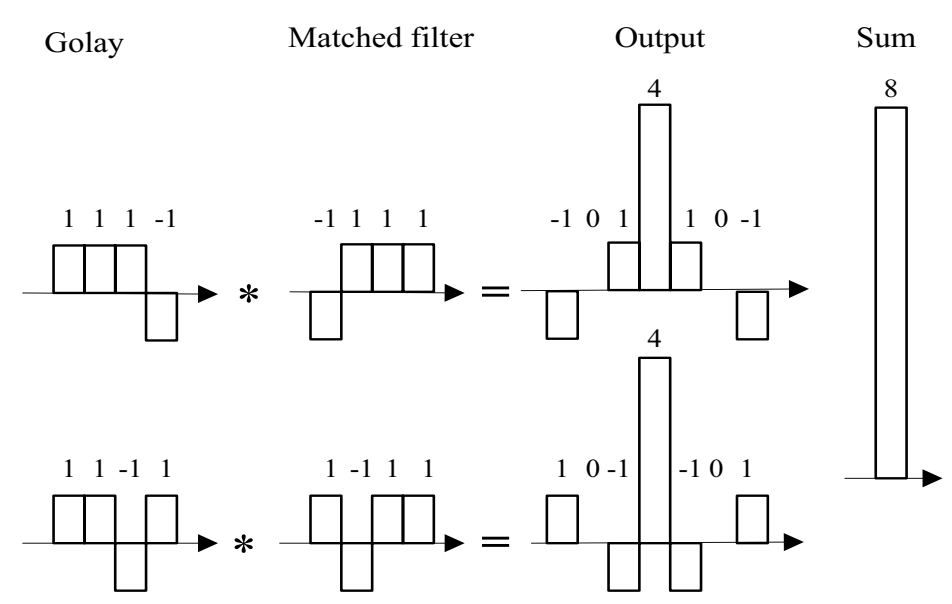

Figure 1. Illustration of decoding process of Golay codes of length 4.

\subsection{Strain estimator}

For a pair of decoded RF echo signals (pre- and post-deformation) of A-line, we first divided the predeformation signal into many partly overlapped windows spacing at a fixed interval. The displacement estimate of a window in pre- deformation signal is the difference of two locations of the window and its matched window in post-deformation signal. The basic algorithm of displacement estimate is the exhaustive search cross-correlation algorithm (ESCCA) which gets the displacement of a predeformation window through seeking the absolute maximum of the cross-correlation function of two pre- and post-deformation windows within the range of post-deformation echo signal. However, ESCCA is not fit to a real-time ultrasound system because of its time-consuming computing. In this paper, we employ phase-zero algorithm [11] (PZA) to estimate displacements, whose main advantage is its computational efficiency without losing accuracy compared with ESCCA. PZA is an iterative algorithm expressed by Pesavento et al. [11] in the form of (4), where $\mathrm{w}_{0}$ is the centre frequency of echo signal, $\mathrm{w}_{\mathrm{c}}$ is the local frequency centroid, $\square \mathrm{t}$ is the window spacing, $\mathrm{T}$ is the window length, $\mathrm{n} \square \mathrm{t}$ is the position of the start of the $n$th window. $x_{1 b}(t)$ and $x_{2 b}(t)$ are the baseband analytic signals of the decoded echo signals $\mathrm{x}_{1}(\mathrm{t})$ and $\mathrm{x}_{2}(\mathrm{t})$, respectively, $\hat{d}_{k, n}$ is the displacement estimate of the nth window at the kth iteration. At the first window, the search is initialized with $\hat{d}_{0,0}=0$. Subsequent searches are each initialized with the final displacement estimate from the previous window, i.e., $\hat{d}_{0, n}=\hat{d}_{K, n-1}$. The number of iterations is usually set to 6 . In practice, we choose $\mathrm{w}_{\mathrm{c}}=\mathrm{w}_{0}$.

$$
\hat{d}_{k+1, n}=\hat{d}_{k, n}+\frac{\arg \left(\exp \left(-j w_{0} \hat{d}_{k, n}\right) \sum_{t=n \Delta t}^{n \Delta t+T} x_{1 b}^{*}(t) x_{2 b}\left(t-\hat{d}_{k, n}\right)\right)}{w_{c}}
$$

It is not generally true that a displacement estimate most closely tracks displacement at the window center. $\mathrm{C}^{\prime}$ espedes and Ophir noted that the actual location is skewed towards sections where the windowed ultrasound signal has high amplitude. This causes artifacts at boundaries between regions of differing echogenicity where the strain estimates are corrupted by unwanted amplitude modulation (AM). We utilize the amplitude modulation correction (AMC) [12] technique to correct these artifacts. The location estimate algorithm using AMC is given as: 


$$
\hat{\boldsymbol{\tau}}_{n}=\frac{\sum_{t=n \Delta t}^{n \Delta t+T} \operatorname{Env}\left\{x_{1 b}(t)\right\} \operatorname{Env}\left\{x_{2 b}\left(t-\hat{d}_{n}\right)\right\} t}{\sum_{t=n \Delta t}^{n \Delta t+T} \operatorname{Env}\left\{x_{1 b}(t)\right\} \operatorname{Env}\left\{x_{2 b}\left(t-\hat{d}_{n}\right)\right\}}
$$

where $\hat{\tau}_{n}$ is the location estimate of the nth window, $\operatorname{Env}\{a\}$ is the envelope of the signal a, and $\hat{d}_{n}$ is the displacement estimate of the nth window.

The strain estimate for the nth window is:

$$
\hat{s}_{n}=\frac{\left(\hat{d}_{n+1}-\hat{d}_{n}\right)}{\left(\hat{\tau}_{n+1}-\hat{\tau}_{n}\right)}
$$

\subsection{SNRe}

One often uses SNRe to evaluate the quality of a strain image. The definition of SNRe:

$$
S N R_{e}=\frac{\mu_{\hat{s}}}{\sigma_{\hat{s}}}
$$

where $\mu_{\hat{s}}$ and $\sigma_{\hat{s}}$ are, respectively, the mean and standard deviation of the strain estimates in a region of uniform elasticity.

\section{Simulation method and results}

\subsection{Simulation method}

RF data of each frame were simulated using Field II [13]. The uniform phantom consists of $1.152 \times 105$ scatterers (fully developed) distributed uniformly throughout $40 \times 36 \times 6 \mathrm{~mm}$ volumes with scattering strengths gaussianly distributed. As the tissue scatterers move mainly along axial direction when the tissue is compressed for strain imaging, we only considered, for simplicity, axial strains which were simulated by rescaling the axial coordinates of the scatterer positions between consecutive frames. Gaussian white noise is added the received waveform. eSNR is measured under the condition of no attenuation by the ratio of the echo signal power of the short pulse to the power of electronic noise. The Golay codes of length 32 are used. The short pulse is a sine wave of two cycles with $5 \mathrm{MHz}$ center frequency. A linear array with 128 transducer elements is used to scan the software phantom. Other fixed parameters include scanning depth $6 \mathrm{~cm}$, attenuation coefficient $0.5 \mathrm{~dB} / \mathrm{cm} / \mathrm{MHZ}$, applied strain $2 \%$, window length $2.4 \mu \mathrm{s}$, and transmit and receive f-number 1.5 .

\subsection{Simulation results}

We first compare the performance of the conventional short pulse and Golay code in eSNR 20dB condition. The strain image in Fig.2a is obtained using the short pulse, which SNRe is 6.02. The strain image in Fig.2b is obtained using the Golay code, which SNRe is 13.03. Therefore, the performance of Golay code is much better than matched filter in eSNR 20dB condition, and the SNRe is improved by $116 \%$. This is because Golay code will increase eSNR by its time-bandwidth-product and the increased eSNR will improve SNRe according to the CRLB theory [14].

We second test the performances of the short pulse and Goaly code in different eSNR conditions. Fig.3 shows that the SNRe is as a function of eSNR. It is anticipated that the Golay code performs much better than the short pulse in low eSNR $(<35 \mathrm{~dB})$ condition. However, it can be also seen that in high eSNR $(>35 \mathrm{~dB})$ condition, the short pulse performs better than Golay code. This is because SNRe 
will nearly cease to increase with eSNR in high eSNR conditions according the CRLB theory [14], and the the range sidelobe level of the Golay code will cause the decorrelation noise which will degrade the accuracy of the displacement estimates.

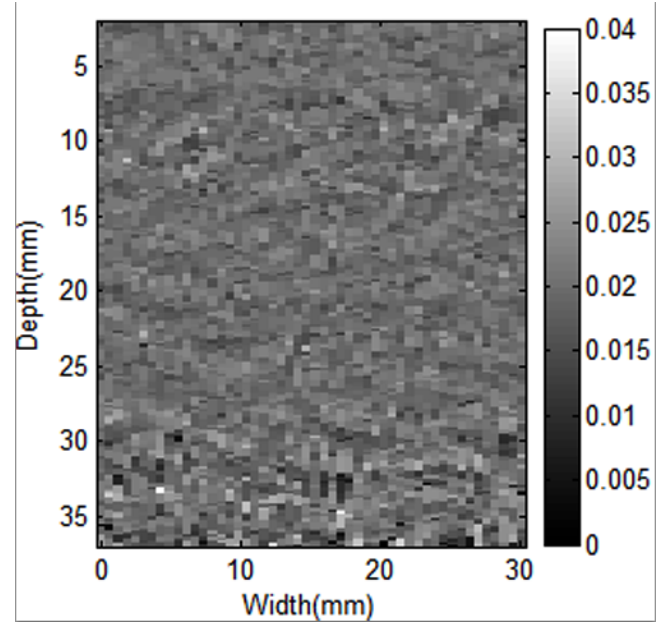

(a) short pulse

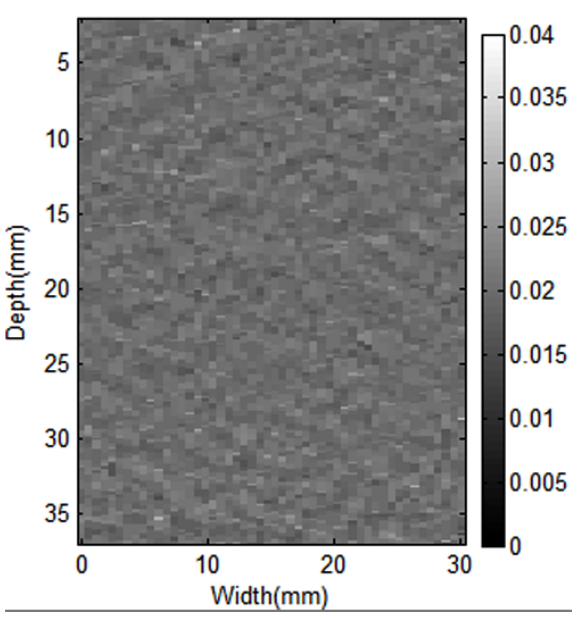

(b) Golay code

Figure 2. (a)A strain image using short pulse in eSNR 20dB. (b)A strain image using Golay code in eSNR 20dB.

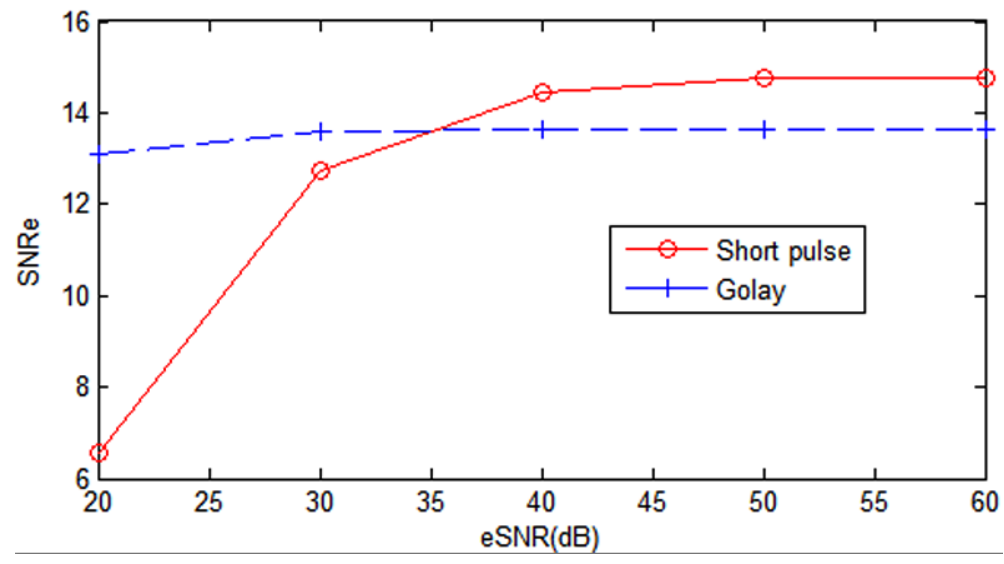

Figure 3. Comparison of short pulse and Golay code in different eSNR conditions.

\section{Conclusions}

Golay code as a code pulse excitation is often used to increase the eSNR in ultrasound imaging. In this paper, we applied Golay code to strain imaging to improve its quality. Instead of being transmitted directly, Golay codes are modulated by the base pulse sequence to achieve better spectral control and greater robustness to motion for multitransmission code. PZA method with AMC is used as strain estimator. We compared the performance of Golay code and the conventional short pulse in simulation method. The simulation results demonstrate that compared with the short pulse, Golay code can improve the SNRe by $116 \%$ in eSNR $20 \mathrm{~dB}$ condition, because the eSNR gain with Golay code increases the accuracy of the displacement estimates. However, in high eSNR conditions, Golay code performs worse than the short pulse, because the performance of Golay code depends mainly on 
its RSLL in high eSNR conditions. Therefore, the optimal conditions for Goaly code to be used in elasticity imaging are the low eSNR, great depth or high attenuation conditions.

\section{Acknowledgments}

This work is in part supported by the Scientific Research Foundation of CUIT (Project No. KYTZ201320), and also in part supported by the Scientific Research Foundation of Education Department of Sichuan Province (Grant No. 14ZB0185).

\section{References}

1. R.G. Barr, K. Nakashima, D. Amy, D. Cosgrove, A. Farrokh, Ultrasound Med. Biol., 41, 1148 (2015)

2. A. Kuzmin, A.M. Zakrzewski, B.W. Anthony, V. Lempitsky, IEEE Trans. Ultrason., Ferroelect., Freq. Contr., 62, 1486( 2015)

3. R. Xia, G. Tao, AK. Thitta, IEEE Trans. Ultrason., Ferroelect., Freq. Contr., 61, 979 (2014)

4. J. Liu, M. F. Insana, IEEE Trans. Ultrason., Ferrolect., Freq. Contr., 52, 231 (2005)

5. H. Peng, D. C. Liu, Biomed. Signal Process. Contr., 8, 130 (2013)

6. H. Peng, D.C. Liu, J. Sichuan Univ. (Eng. Sci. Ed.), 44, 127 (2012)

7. M. O’Donnell, IEEE Trans. Ultrason., Ferroelec., Freq. Contr., 39,341(1992)

8. T. Misaridis, Ultrasound Imaging Using Coded Signals (Ph.D. thesis, Technical University of Denmark, 2001)

9. R. Y. Chiao, X. Hao, IEEE Trans. Ultrason., Ferroelect., Freq. Contr., 52, 160 (2005)

10. C.Leavens, R. Williams, F.S. Foster, S. Member, P.N. Burns, M.D.Sherar, IEEE Trans. Ultrason. Ferroelectr. Freq. Control, 54, 2082 (2007)

11. A. Pesavento, C. Perrey, M. Krueger, H. Ermert, IEEE Trans. Ultrason., Ferroelect., Freq. Contr., 46, 1057 (1999)

12. J. E. Lindop, G. M. Treece, A. H. Gee, R. W. Prager, IEEE Trans. Ultrason., Ferroelect., Freq. Contr., 54, 1751 (2007)

13. J. A. Jensen, Proc. 10th Nordic-Baltic Conf. Biomed. Imag., 4, 351 (1996)

14. W.F. Walker, G.E. Trahey, IEEE Trans. Ultrason., Ferroelect., Freq. Contr., 42, 301 (1995) 